\title{
Cardiac Surgery Conduct during COVID-19 Pandemic
}

\author{
Manoj Kumar Sahu ${ }^{1}$ Prateek Vaswani ${ }^{2}$ Amitabh Satsangi ${ }^{2}$ Sarvesh Pal Singh ${ }^{1}$ \\ Palleti Rajashekar ${ }^{2}$ Velayoudam Devagourou² Shiv Kumar Choudhary²
}

\footnotetext{
${ }^{1}$ Intensive Care for Cardiothoracic and Vascular Surgery, CT Centre, All India Institute of Medical Sciences, Ansari Nagar, New Delhi, India

2Department of Cardiothoracic and Vascular Surgery, All India Institute of Medical Sciences, Ansari Nagar, New Delhi, India

J Card Crit Care:2020;4:91-95
}

\author{
Address for correspondence Manoj K Sahu MD, DNB, Intensive \\ Care for CTVS, CTVS office, 7th floor, \\ CN Centre, All India Institute of Medical Sciences, New Delhi, \\ 110029, India (e-mail: drmanojsahu@gmail.com).
}

\begin{abstract}
Background Health care is seriously affected by the coronavirus disease 2019 (COVID-19) pandemic with alarming effects upon conduct of cardiac surgery. The initial resource conservation strategy has to modify for handling the surging case load due to deference of routine care in the face of pandemic.

Methods The cardiac surgical practice during the lockdown period (from 25th march till 25th June) at a tertiary care centre was observed. The cardiac diagnosis of the ones operated, conduct algorithm, and working policy were analyzed. Descriptive statistics was applied to calculate the percentages of different case subsets in both adult and pediatric groups.

Results A total of 93 cardiac patients were consecutively operated during the 3 months' period in two cardiac theatres of a total eight dedicated and were rotated cyclically. A total of 37 (39.78\%) adult cardiac surgeries were performed out of 93 cases, with coronary artery bypass grafting (11.83\%: 11/93) and valvular heart diseases (11.83\%: 11/93) constituting the majority. Pediatric cardiac surgeries constituted 56 cases $(60.21 \%)$ which comprised of arterial switch operation (19.35\%), total anomalous pulmonary venous connection (8.60\%), and Blalock Taussig shunts (7.53\%) predominantly. There was no COVID-19-related mortality and none of the health-care workers developed COVID-19 in the entire study period.

Keywords

- COVID-19 pandemic

- cardiac surgery

- OR and ICU

preparedness

Conclusions The initial phase of resource conservation has undermined the routine cardiac surgical practice. The study showed that strict adherence to management algorithm is necessary for persisting smooth continuation of cardiac surgical practice with provision of optimum critical care. The strategic comeback against COVID-19 would urge institutional development of protocols to aid the post-surge period.
\end{abstract}

\section{Introduction}

Coronavirus disease 2019 (COVID-19) has established itself as a global pandemic, affecting economy and health-care practices worldwide. The pandemic has affected more than 200 countries around the world including India. ${ }^{1}$ Globally, it has escalated rampantly paralyzing the health-care services.

published online November 3, 2020
DOI https://doi.org/ $10.1055 / \mathrm{s}-0040-1721183$ ISSN 2457-0206.
On March 24, 2020, the Government of India ordered a nationwide lockdown for 21 days, limiting movement of the entire 1.3 billion population as a preventive measure against the COVID-19 pandemic in the country. ${ }^{2}$ This was enforced in phases with current being phase 6 or unlock 2 . The emphasis on conservation of critical care resources with suspension of elective surgical procedures was implemented in liaison with 
this mitigation strategy to prepare for the upcoming surge. COVID-19 has caused a deep impact on cardiac surgical practices by limiting the staff, intensive care unit (ICU) beds, ventilation and monitoring sites, and other resources required for an effective conduct. The impact of COVID-19 on cardiac surgery at our center has been profound. Cardiovascular diseases are leading cause of mortality in the world. ${ }^{3}$ Though cardiovascular diseases can be avoided for a period of time, they cannot be completely ignored as the burden of disease is massive in the society and complete withdrawal of surgical services is not an adequate answer. Amid the pandemic, safeguard of critical resources has been regarded as the prime matter of concern. Although delaying elective surgeries is feasible, the emergency cases require early intervention to avoid grave sequelae. Therefore, this study was conducted in our center to see the various cardiac surgical procedures being catered to amid this jeopardized medical crisis with attention to the challenges faced, which may guide the pathway for a strategic comeback.

\section{Methodology}

A cross-sectional study was performed in cardiothoracic and vascular surgery department of a tertiary care center over a 3-month period beginning from March 25, 2020, when nationwide lockdown was enforced till June 25 as time frame. The admission policy, testing algorithm, comprehensive cardiac diagnosis, operating room (OR), and ICU preparedness strategy were studied. The cases performed were divided into adult and pediatric. Similar preparations were done for ICU and ORs with preparation of plans to mitigate the crisis. These have been elaborated and discussed.

Elective cases were suspended from the first lockdown period, the outpatient services were stopped, and cardiac surgical cases were triaged from this time onwards. About 50\% of cardiac surgical resources and workforce were dedicated to COVID-19 facility. All cardiac surgical cases were stratified to emergency, urgent, and elective cases for efficient triaging. All patients underwent reverse transcriptase polymerase chain reaction (RT-PCR) testing following which the further

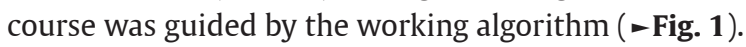

Emergency cases-They were defined in adult and pediatric as those who were to be operated within 24 to 48 hours and any delay would cause significant impact on mortality.

Urgent cases-These were defined as those cases that needed surgical intervention within 1 to 2 weeks provided the resources were available, and waiting further would adversely affect the mortality or morbidity.

Elective cases-These were the cases that could be deferred till pandemic surge started subsiding.

The cases were being triaged in multidisciplinary meetings considering the cardiac diagnosis, usual recovery period with any unexpected postoperative course. This included the cardiologist, anesthesiologist, intensivis, and cardiac surgeons.

Statistical analysis-All adult and pediatric patients were stratified according to the disease and surgical procedure, descriptive analysis was done, and number and percentage of different cases in both categories were determined.

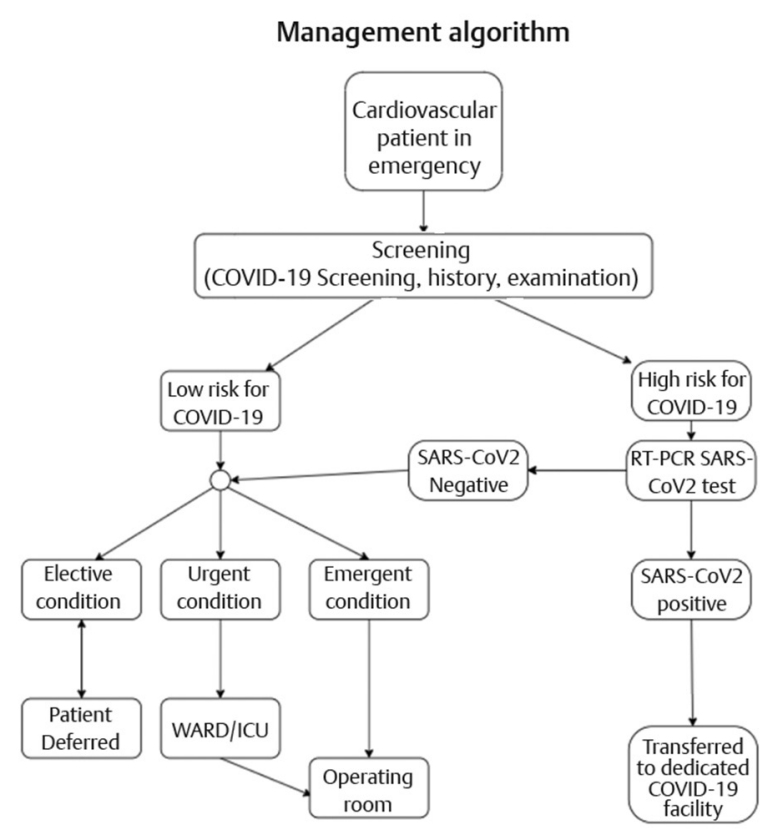

Fig. 1 The working algorithm. COVID-19, coronavirus disease 2019; ICU, intensive care unit; RT-PCR, reverse transcriptase polymerase chain reaction; SARS-CoV2, severe acute respiratory syndrome coronavirus 2.

Table 1 Adult cardiac surgeries (total $=37$ )

\begin{tabular}{|l|l|l|l|}
\hline $\begin{array}{l}\text { S. } \\
\text { No. }\end{array}$ & Operative surgery & $\begin{array}{l}\text { Total number } \\
\text { of cases } \\
(\boldsymbol{n}=\mathbf{3 7}) \mathbf{( \% )}\end{array}$ & Remarks \\
\hline $\mathbf{1}$ & $\begin{array}{l}\text { Coronary artery bypass } \\
\text { grafting }\end{array}$ & $11(11.8)$ & $\begin{array}{l}\text { Emergency 1, } \\
\text { urgent 10 }\end{array}$ \\
\hline $\mathbf{2}$ & Valve surgeries & $11(11.8)$ & $\begin{array}{l}\text { Emergency 6, } \\
\text { urgent 5 }\end{array}$ \\
\hline $\mathbf{3}$ & Aortic aneurysm repair & $6(6.45)$ & $\begin{array}{l}\text { Emergency 2, } \\
\text { urgent 4 }\end{array}$ \\
\hline $\mathbf{4}$ & Aortic dissection & $3(3.22)$ & Emergency \\
\hline $\mathbf{5}$ & $\begin{array}{l}\text { Pulmonary thromboen- } \\
\text { darterectomy }\end{array}$ & $1(1.07)$ & Urgent \\
\hline $\mathbf{6}$ & $\begin{array}{l}\text { Left atrial myxoma } \\
\text { excision }\end{array}$ & $1(1.07)$ & Urgent \\
\hline $\mathbf{7}$ & $\begin{array}{l}\text { Left carotid body tumor } \\
\text { excision }\end{array}$ & $1(1.07)$ & Urgent \\
\hline $\mathbf{8}$ & $\begin{array}{l}\text { Pacemaker generator } \\
\text { extraction }\end{array}$ & $1(1.07)$ & Urgent \\
\hline $\mathbf{9}$ & $\begin{array}{l}\text { Brachial } \\
\text { thromboembolectomy }\end{array}$ & $1(1.07)$ & Emergency \\
\hline $\mathbf{1 1}$ & Carotid aneurysm repair & $1(1.07)$ & Emergency \\
\hline
\end{tabular}

\section{Results}

A total of 93 cases were operated in cardiothoracic and vascular center at our institute in 3 months from March 25, 2020 to June 25,2020 . There were 37 adults and 56 pediatric cases as mentioned in - Tables $\mathbf{1}$ and $\mathbf{2}$, respectively. Out of the eight designated ORs for cardiothoracic and vascular surgeries, only two were functional in 1 day with the rest six being closed. There was cyclical rotation of ORs. 
Table 2 Pediatric cardiac surgeries (total $=56$ cases)

\begin{tabular}{|c|c|c|c|}
\hline $\begin{array}{l}\text { S. } \\
\text { No. }\end{array}$ & Operative procedure & $\begin{array}{l}\text { Total number } \\
\text { of cases } \\
(n=56)(\%)\end{array}$ & Remarks \\
\hline 1 & ASO & $18(19.3)$ & $\begin{array}{l}\text { Emergency in } \\
10 \text { cases with } \\
\text { intact ventricu- } \\
\text { lar septum, } \\
\text { urgent in } 6 \text { VSD } \\
\text { cases, urgent } \\
2 \text { cases with } \\
\text { ASD }\end{array}$ \\
\hline 2 & TAPVC repair & $8(8.60)$ & $\begin{array}{l}\text { Emergency in } \\
5 \text { cases, urgent } \\
\text { in } 3 \text { cases }\end{array}$ \\
\hline 3 & BT shunt & $7(7.5)$ & Emergency \\
\hline 4 & TOF repair & $5(5.38)$ & $\begin{array}{l}\text { Emergency in } \\
3 \text { cases, urgent } \\
\text { in } 2 \text { cases }\end{array}$ \\
\hline 5 & PA banding & $3(3.2)$ & Urgent \\
\hline 6 & VSD closure & $3(3.2)$ & Urgent \\
\hline 7 & $\begin{array}{l}\text { Epicardial pacemaker } \\
\text { insertion }\end{array}$ & $2(2.15)$ & Urgent \\
\hline 8 & Diaphragmatic plication & $2(2.15)$ & Urgent \\
\hline 9 & $\begin{array}{l}\text { Aortic valve replacement } \\
\text { with ruptured sinus of } \\
\text { Valsalva repair }\end{array}$ & $1(1.07)$ & Urgent \\
\hline 10 & Senning operation & $1(1.07)$ & Urgent \\
\hline 11 & $\begin{array}{l}\text { Complete atrioventricular } \\
\text { septal defect repair }\end{array}$ & $1(1.07)$ & Urgent \\
\hline 12 & $\begin{array}{l}\text { Bidirectional Glenn + left } \\
\text { PA plasty }\end{array}$ & $1(1.07)$ & Urgent \\
\hline 13 & $\begin{array}{l}\text { TV vegetectomy + TV } \\
\text { replacement }\end{array}$ & $1(1.07)$ & Urgent \\
\hline 14 & $\begin{array}{l}\text { Left thoracotomy and } \\
\text { pleural drainage }\end{array}$ & $1(1.07)$ & Urgent \\
\hline 15 & Mediastinal mass excision & $1(1.07)$ & Urgent \\
\hline 16 & Left lung decortication & $1(1.07)$ & Urgent \\
\hline
\end{tabular}

Abbreviations: ASD, atrial septal defect; ASO, arterial switch operation; BT, Blalock Taussig; PA, pulmonary artery; TAPVC, total anomalous pulmonary venous connection; TOF, Tetralogy of Fallot; TV, tricuspid valve; VSD, ventricular septal defect.

We ensured all patients underwent RT-PCR test for COVID-19 before being taken for surgery prior to admission; however, it could not be done in one patient who was a 62 -year-old male with acute coronary syndrome following failed percutaneous transluminal coronary angioplasty (PTCA) taken up for emergency coronary artery bypass grafting. None of the patients were proven COVID-19 positive at the time of surgery; however, adequate precautions were taken in all.

There was a 2-month-old child who was admitted postballoon atrial septostomy transferred from cardiology side and post-admission turned out to be COVID-19 positive. The child was isolated in COVID-19 designated area with the mother who was also COVID-19 positive in the hospital and was taken for surgery when turned negative. The adult male patient was subjected to RT-PCR with endotracheal secretions in postoperative period that revealed negative result.
The postoperative patients were constantly monitored for symptom development and chest X-rays were done routinely for any new findings. There were four patients ( 2 adults and 2 pediatric patients) who became COVID-19 positive in the postoperative period. These patients were transferred to dedicated COVID-19 facility in the hospital for management, and all four recovered from COVID-19 and were transferred back after negative RT-PCR report before they were discharged.

Five patients (5.38\%) expired in the 3-month study period. Two were post-obstructed supracardiac total anomalous pulmonary venous connection (TAPVC) repair cases with severe pulmonary artery hypertension, managed with delayed sternal closure and inhaled nitric oxide, but they subsequently developed sepsis with multiorgan dysfunction syndrome (MODS) before they succumbed. Third patient post-arterial switch with integrated extracorporeal membrane oxygenator (ECMO) support could not be weaned off due to severe biventricular dysfunction, but the patient subsequently developed sepsis that was managed with appropriate antibiotics but succumbed due to malignant ventricular arrhythmia post-ECMO decannulation. Fourth patient of post-BT (modified Blalock Taussig) shunt developed severe pulmonary hemorrhage and subsequently expired. Fifth patient post-Senning was managed with delayed sternal closure due to severe biventricular dysfunction and atrial arrhythmias, but subsequently developed sepsis with multiorgan dysfunction syndrome before the child succumbed. None of the patients in this study cohort developed any neurological deficit.

There was no observed COVID-19-related morbidity or mortality. During the entire study period, none of the health-care personnel involved tested positive.

\section{Discussion}

The phasic transition in lockdown has been accompanied with phasic shift in hospital strategy for reallocation of limited critical care resources for those in dire need. The number of cardiac surgeries has witnessed a significant decline since the beginning of the lockdown that was imposed from March 24,2020 . The number of cases declined significantly compared with prepandemic times. The average cases being conducted at our center are $\sim 300$ per month. This signified $\sim 90 \%$ decline (93 cases in 3 months) in COVID-19 times with only emergency or urgent cases being catered. Similarly, in Italy's Lombardy region, 16 of 20 cardiac surgical units discontinued cardiac operations with the remaining four units forming the hub for closed units. ${ }^{4}$ In many US states including New York, all nonemergency cardiac surgeries were suspended early in response to the rising numbers of infected population with COVID-19. ${ }^{5}$ We also suspended all outpatient services and elective cardiac surgical procedures for conserving critical resources; however, conduct of emergency cases were performed. There are grave complications with effects on mortality and morbidity on delaying cardiac surgical procedures. ${ }^{6,7}$ The multidisciplinary meeting among cardiologist, cardiac surgeons, anesthesiologists, and intensive care experts was responsible for priority list formulation, 
which formed the basis of our conduct pathway. Similar recommendations for triaging patients and critical resource utilization during this pandemic crisis have been proposed appropriately for adult cases, ${ }^{8-10}$ while the study by Stephens et al described the urgent and emergency cases for pediatric cardiac surgeries. ${ }^{9}$

The postoperative valvular heart diseases with prosthetic heart valve thrombosis following failed medical thrombolysis with New York Heart Association grades III/IV, the primary mitral valve pathologies with large left atrial clot likely to embolize, and multivalvular pathologies presenting in heart failure even on optimum medical therapy constituted the $11.8 \%$ (11/93). Coronary artery disease with triple vessel involvement with ongoing chest pain, failed emergency PTCA in acute coronary syndrome or left main involvement, and other lesions nonamenable to PTCA according to cardiologist opinion constituted $11.8 \%$ (11/93) of the cases. The other adult cases are mentioned in - Table $\mathbf{1}$ for reference with description of them being emergency or urgent status. The pediatric cases comprised majority of arterial switch operations in 19.3\% (18/93), TAPVC repair in 8.60\% (8/93), and BT shunts for children with profound cyanosis with history of cyanotic spells in $7.53 \%$ (7/93). Five patients $(5.38 \%, 5 / 93)$ were Tetralogy of Fallot, of which three had significant history of cyanotic spells and two were symptomatic with poor weight gain and breathlessness with optimum medical therapy. Three patients with large ventricular septal defect with failure to thrive and recurrent lower respiratory tract infection (LRTI) requiring hospital admissions were also admitted in view of failure partially managed on medical therapy after COVID-19 testing and underwent surgery with uneventful postoperative period. Two patients came with LRTI and were admitted on pediatric cardiology side after being tested COVID-19 negative; they required mechanical ventilation due to severe pulmonary artery hypertension, which required pulmonary artery banding as an urgent intervention. Postoperatively, they were weaned off with one of them requiring tracheostomy in view of prolonged mechanical ventilation and other was successfully extubated. Rest cases are mentioned in - Table 2 for reference. None of the emergency cases turned out to be COVID-19 positive; so, in this study there is no experience with surgery of COVID-19-positive patient. The patients who turned out be COVID-19 positive by RT-PCR were managed as discussed above.

Designated areas for donning and doffing were created with proper supervision by infection control staff. Practice drills in OR and ICU were performed to effectively handle crisis situations that included minimizing aerosol generating procedures (AGPs), wearing facial shields, selection of most skilled member as team leader taking responsibility, and practicing safe cardiopulmonary resuscitation.

A minimal necessary personnel approach was followed while transferring patient to and from the OR, during induction and surgery, and while carrying out other AGPs in the OR or ICU. The ORs were well ventilated with good light source with provisions for viral filter circuits, intubation box, video-laryngoscope, and appropriate monitors. The OR gas exchanges per hour were maintained according to institutional recommendations. The negative pressure rooms, however, were not available. Transesophageal echocardiography was not done in the OR in this period as a precautionary measure. Cyclical utilization of ORs with only two of eight being functional in 1 day was done. The surgeons were allowed to enter the OR after a stipulated time period post-induction to avoid crowd. The most skilled members performed surgery minimizing exposure time with minimal necessary personnel scrubbed and practiced meticulous hemostasis for avoiding re-explorations. Post-surgery, the instruments were being handled with utmost precautions by nursing personnel carrying out cleaning with autoclaving under supervision after donning appropriate personal protective equipment. The ORs were cleaned with disinfectants and fumigated post-procedure. The next two ORs were made available for the next day. In ICU, social distance norms with 6 feet distance between adjacent beds were followed with designated staff. The observers in anesthesia, cardiac surgery, and intensive care were not accepted during this period. Visitors and patient's attendants were not allowed in the ICU. Telecommunication with video calling was followed with the relatives or guardians except in pediatric patients where mothers were allowed to enter the ICU once or twice daily for expressed milk for enteral feeding of these infants. The parents of pediatric cases especially mothers were evaluated with COVID-19 RT-PCR test before admission and the same person was allowed to enter. Early ambulation was performed and a low threshold was kept for repeat testing in cases of clinical doubt. The strict vigilance helped us to detect four patients who were tested positive in the postoperative period who were managed conservatively in COVID-19 designated areas in the hospital.

Teleconferencing formed the basis of OPD follow-up in the discharged patients. This was explained to patients and their attendants before admission in the facility. Education on multimedia communication, image exchange on messengers, exchange of reports, change of medications, calling for emergency visit, inquiring wound status, and other queries was performed.

The study also showed that by strict adherence to the protocols, health-care personnel were efficiently protected, with none of them contracting this contagious disease. The overall study mortality rate was 5.38\% (5/93) with no COVID-19 related mortality that signifies the success of institutional mitigation strategy. In spite of negative preoperative tests, four patients turned COVID-19 positive, which highlights the false negative rates of the RT-PCR test. This has been reported to the tune of 30\%. This also underscores the importance of a strict vigilance for postoperative development of symptoms with a low threshold for repeat testing. The social distancing between adjacent beds prevented transfer of infection to other patients due to contacts who were tested as per the institutional infectious disease surveillance program team. The study results indicate that utmost level of care can be 
provided with optimal outcomes while maintaining critical care resources in crisis times. This would also help in re-establishment of routine procedures once the pandemic starts to decline.

\section{Conclusion}

COVID-19 has challenged the medical specialities, driving the routine care into a jeopardy. The critical resource preservation has to be balanced with provision of emergency care with optimum critical care standards to the ones in dire need. The herculean task of carrying out safe cardiac surgery seems plausible with strict adherence to institutional protocols with no added risk to either health-care professionals or the patients. The return to regular course from this unplanned detour seems mandatory for avoiding an avoidable crisis.

\section{Funding}

None.

\section{Conflict of Interest}

None.

\section{References}

1 Worldometer. COVID-19 coronavirus pandemic. Available from: https://www.worldometers.info/. Accessed July 20, 2020

2 CoronavirusinIndia:ModiOrdersTotalLockdownof21Days-The New York Times [Internet]. [cited 2020 Jun 29]. Available from: https://www.nytimes.com/2020/03/24/world/asia/indiacoronavirus-lockdown.html. Accessed Aug 31, 2020

3 The top 10 causes of death. [cited 2020 Jun 28]. Available from: https://www.who.int/news-room/fact-sheets/detail/the-top10-causes-of-death. Accessed Aug 31, 2020

4 Bonalumi G, di Mauro M, Garatti A, Barili F, Gerosa G, Parolari A; Italian Society for Cardiac Surgery Task Force on COVID-19 Pandemic. The COVID-19 outbreak and its impact on hospitals in Italy: the model of cardiac surgery. Eur J Cardiothorac Surg 2020;57(6):1025-1028

5 Centers for Medicare \& Medicaid Services. Non-emergent, elective medical services, and treatment recommendations. Available from: https://www.cms.gov/files/ document/31820cms-adult-elective-surgery-and- procedures-recommendations. pdf. Accessed April 21, 2020

6 Malaisrie SC, McDonald E, Kruse J, et al. Mortality while waiting for aortic valve replacement. Ann Thorac Surg 2014;98(5): 1564-1570, discussion 1570-1571

7 Head SJ, da Costa BR, Beumer B, et al. Adverse events while awaiting myocardial revascularization: a systematic review and meta-analysis. Eur J Cardiothorac Surg 2017;52(2):206-217

8 Hassan A, Arora RC, Lother SA, et al; of the Canadian Society of Cardiac Surgeons. Ramping up the delivery of cardiac surgery during the covid-19 pandemic: a guidance statement from the Canadian Society of Cardiac Surgeons. Can J Cardiol 2020;36(7):1139-1143

9 Stephens EH, Dearani JA, Guleserian KJ, et al. COVID-19: crisis management in congenital heart surgery. Ann Thorac Surg 2020;110(2):701-706

10 Haft JW, Atluri P, Ailawadi G, et al; Society of Thoracic Surgeons COVID-19 Task Force and the Workforce for Adult Cardiac and Vascular Surgery. Adult cardiac surgery during the COVID-19 pandemic: a tiered patient triage guidance statement. Ann Thorac Surg 2020;110(2):697-700 